\title{
Videofluroscopic Assessment of Dysphagia in Patients with Minimal Associated Pathological Vocal Fold Lesions
}

\author{
Original \\ Article \\ Hassan Hosny Ghandour, Yara Hany Hadhoud, Yomna Hassan ElFiky \\ Department of Otorhinolaryngology, Faculty of Medicine, Ain Shams University, Cairo, \\ Egypt
}

\begin{abstract}
Background: Swallowing difficulties to variable consistencies is an alarming sign.

Objective: The aim of this study is to investigate symptoms of Dysphagia reported by patients with Minimal Associated Pathological vocal fold lesions (MAPLs) on basis of subjective as well as objective measures in patients with dysphonia. Patients and Methods: This study was applied on 50 patients with age ranging from 15-50 years diagnosed as being dysphonic secondary to Minimal Associated Pathological vocal fold lesions confirmed by objective and clinical measures. Results: After the application of the A-EAT-10 questionnaire on 50 dysphonic patients who were selected to participate in this study, 12 cases (about 24\%) were considered dysphagic with a score above 3. It was necessary to search for the cause of dysphagia by an objective method. So, patients reported as dysphagic by the A-EAT-10 underwent Videofluoroscopic examination. Within these 12 patients, none of them $(0 \%)$ exhibited any signs of swallowing abnormalities detected during VFSS, to justify the reported dysphagia. Thus, there was no correlation between the presence of dysphagia reported by the A-EAT and the videofluoroscopic findings.

Conclusion: : Symptoms of dysphagia were reported by patients with minimal associated pathological lesions and were documented with a screening tool like the A-EAT. However, that was not confirmed by a videofluoroscopic examination.
\end{abstract}

Key Words: Dysphagia, minimal associated pathological vocal fold lesions, video videofluoroscopy

Received: 22 July 2021, Accepted: 03 September 2021

Corresponding Author: Yomna Elfiky, Department of Otorhinolaryngology Faculty of Medicine Ain Shams University, Cairo, Egypt, Tel.: 01111889805, E-mail: yomna@med.asu.edu.eg

ISSN: 2090-0740, 2021

\section{INTRODUCTION}

Oropharyngeal dysphagia may be due to morphological abnormalities, neurological disorders whereas esophageal dysphagia may be due to structural abnormalities, inflammation and esophageal dysmotility ${ }^{[1]}$.

Symptoms include biting or chewing difficulties preventing bolus formation, affected range of movement of the tongue and/or weakness of the tongue that requires extra time to get the bolus ready for swallowing, drooling, food pocketing in the lateral sulcus and sometimes aspiration before the swallow ${ }^{[2]}$.

Minimal Associated Pathological Lesions "MAPLs" such as Polyps, nodules, cysts, Reinke's edema, polypoid degeneration and contact granuloma are examples of these non-inflammatory, benign lesions. The most common risk factors include extroverts with a talkative personality and occupations with high vocal demands. Other factors that can potentiate vibratory injury include smoking, acid reflux, uncontrolled allergies, and infections ${ }^{[3]}$. Assessment includes history-taking, endoscopic examination and imaging studies if needed to detect the exact cause.
Subjective tools focus on the patients' quality of life, emotional and psychosocial aspects. One of these screening approaches ${ }^{[4]}$ is the Arabic version of the Eating Evaluation Tool (A-EAT-10).

Impaired swallowing that is regularly masked by the phonatory complaint in patients with minimal associated pathological lesions is not widely investigated previously. It is a common understanding that these patients have hyperfunctional laryngeal behavior, which hastens the stress at the mid-membranous part of the vocal folds, this might also disturb one or extra of the kinematics of swallowing ${ }^{[4]}$. In these patients, dysphagia might also be a frequent symptom that is often ignored although being reported by screening tools. These suggested mechanisms remain hypothetical in the absence of videofluoroscopic or manometric studies of swallowing ${ }^{[4]}$.

\section{PATIENTS AND METHODS}

\subsection{Study design:}

The study was a cross-sectional study, applied on patients diagnosed as being dysphonic, secondary to non- 
neoplastic vocal fold lesions and confirmed objective and clinical measures. Attending at the Phoniatric outpatient hospital at the period from November 2018 to May 2019.

\subsection{Subjects:}

50 patients with age ranging from $15-50$ years diagnosed as being dysphonic secondary to minimal associated pathological lesions. These patients attended the Phoniatric outpatient clinic. A convenience sample was used to select the cases upon the following inclusion and exclusion criteria.

\section{Inclusion Criteria:}

- Age ranging from 15 to 50 years.

- Patients being diagnosed by clinical and objective tests as being dysphonic secondary to non-neoplastic vocal fold lesions.

\section{Exclusion criteria:}

- Age below 15 years or above 50 years.

- Patients with recent history of respiratory tract infection, laryngeal surgery or manipulation, neurologic disorders, head and neck tumors, history of chemotherapy/ radiotherapy to head and neck, or any other proved cause of dysphagia.

\subsection{All patients have been subjected to the following:}

I- Elementary Diagnostic Procedures including: Patient interview and cautious history taking.

\section{II- Clinical Diagnostic Aids:}

a- Augmentation and documentation of the glottic picture using: Telescopic rigid fiberopticorolaryngoscopy or Flexible nasolaryngoscopy.

b- The Arabic Eating Assessment Tool-10 (A-EAT-10) as a major screening measure for dysphagia. Patients with a rating above three will be viewed to have dysphagia.

The Arabic Eating Assessment Tool-10 (A- EAT-10) (see appendix):

The Arabic EAT-10 is a self-administered, symptomspecific screening. It is a noninvasive tool to measure patients' perception of their swallowing problems, for the subjective assessment of dysphagia in an Arabic talking population. It is an administered questionnaire; it takes solely two minutes to be achieved and wants no training. It consists of 10 statements in which the affected person evaluates his/her perceived degree of a swallowing problem $^{[4]}$.

\section{Scoring and ranking interpretation:}

Rating the problem is done on a scale of $0-4(0=$ no problem, $4=$ severe problem). The clinician only adds the numbers. The normative data revealed that the A-EAT-10 rating of higher than three is viewed dysphagia. An increased EAT-10 rating shows a higher self-perception of dysphagia.

c- Videoflouroscopic swallowing study (VFSS):

- Personell involved: The phoniatrician, radiologist and a radiologytechnologist.

- Patient setting: The patient could be seated, reclined on a stretcher,or standing and observed from a lateral view to allow for the documentation of bolus flow and structural movement. Depending on the course of the evaluation, it might be necessary to observe the patient in an anteroposterior view as well.

- Bolus consistency: Using a standard protocol with each patient is ideal. Deviation can occur at any time in the protocol if patient safety is of concern. In most protocols, various volumes of thin liquid barium are administered along with barium pudding and a barium-coated solid.

- Bolus Volume: The examination is generally initiated with small thin liquid volumes $(5 \mathrm{~mL})$ then larger liquid volumes (10 and $20 \mathrm{~mL})$ and continuous cup drinking, semisolids (one tea spoon), and solids. Volumes and consistencies are typically administered twice to reliably judge swallowing function. Ideally, the patient selfadministers all volumes and consistencies, and no cuesto swallow are provided. The VFSS was recorded for later observation. Careful reviewing of the examination using slow motion and frame-by-frame analysis is critical as many events are occurring simultaneously or with inmilliseconds of each other.

- Possible abnormal findings: Delayed Triggering of swallowing reflex, premature spillage, absence of swallowing reflex, multiple swallows to the bolus, residue in vallecula and/or pyriform fossae, penetration and aspiration.

\section{Data Management and Analysis:}

The collected data was revised, coded, tabulated and introduced to a PC, using Statistical package for Social Science (SPSS27). Data was presented and suitable analysis was done according to the type of data obtained for each parameter.

\section{Descriptive statistics:}

1. Mean and Standard deviation $( \pm \mathrm{SD})$ for numerical data.

2. Frequency and percentage of non-numerical data.

\section{RESULTS}

\subsection{Demographic data for cases:}

Among the cases, the patients' age ranged from 15 to 50 years with a mean of $35.74 \pm 8.724$ years as shown in Table 1. Regards the gender, there were 23 males (46\%) and 27 females (54\%) as shown in Table 2. 
Table 1: Mean age among studied group

\begin{tabular}{lccc} 
& Minimum & Maximum & Mean \\
\hline Age in years & 15 & 50 & 35.74 \\
\hline
\end{tabular}

Table 2: Demographic data for cases

\begin{tabular}{lccc}
\hline & & Frequency & Percent \\
\hline \multirow{3}{*}{ Gender } & Female & 27 & $54.0 \%$ \\
& Male & 23 & $46.0 \%$ \\
\hline
\end{tabular}

\subsection{The frequency and percentage of dysphagia by the Arabic EAT-10}

In the current study, the most common vocal fold lesion in patients with dysphonia was vocal fold polyp. The frequency and percentage of all vocal fold lesions included are shown in Table 3.

4.3. Frequency of dysphagia by the Arabic EAT10 and frequency of swallowing abnormalities by Videoflouroscopy:

In 50 patients with dysphonia, $24.0 \%$ had dysphagia as evidenced by the Arabic EAT-10 score of above three as shown in Table 4.

Frequency of swallowing abnormalities by videoflouroscopy in the studied cases who were found dysphagic by the A-EAT-10 was Zero as shown in Table 5.

4.4. Correlation between frequency of Dysphagia reported by the Arabic EAT-10 and swallowing abnormalities detected by Videoflouroscopy:

There is a difference in frequency of reported dysphagia by the Arabic EAT-10 and the swallowing abnormalities detected by videoflouorocopy, being $24 \%$ by the Arabic EAT-10 with different frequency distribution among different vocal fold pathologies and Zero by Videoflouroscopy whatever the lesion is. This discrepancy indicates no correlation between both frequencies as shown in Figure 1.

Table 3: The frequency and percentage of each vocal fold lesion in participating patients

\begin{tabular}{lcc}
\hline Vocal Fold lesion & Frequency & Percent \\
\hline Vocal Fold Polyp & 17 & $34.0 \%$ \\
Vocal Fold Cyst & 6 & $12.0 \%$ \\
Vocal Fold Nodules & 14 & $28.0 \%$ \\
Reinke's Edema & 5 & $10.0 \%$ \\
Polypoid Degeneration & 4 & $8.0 \%$ \\
Contact Granuloma & 4 & $8.0 \%$ \\
Total & 50 & $100.0 \%$ \\
\hline
\end{tabular}

Table 4: The frequency and percentage of dysphagia by the Arabic EAT-10 in all participating patients

\begin{tabular}{lccc}
\hline & & Frequency & Percent \\
\hline & (Positive(score $>3$ & 12 & $24.0 \%$ \\
Dysphagia by the A-EAT-10 & (Negative(score $\leq 3$ & 38 & $76.0 \%$ \\
& Total & 50 & $100.0 \%$ \\
\hline
\end{tabular}


Table 5: The frequency and percentage of swallowing abnormalities by Videoflouroscopy in dysphagic patients by the A-EAT-10

\begin{tabular}{|c|c|c|c|}
\hline & & Frequency & Percent \\
\hline \multirow{2}{*}{ Swallowing abnormalities by Videoflouroscopy } & Positive & 0 & $0 \%$ \\
\hline & Negative & $\%$ & 100.0 \\
\hline
\end{tabular}

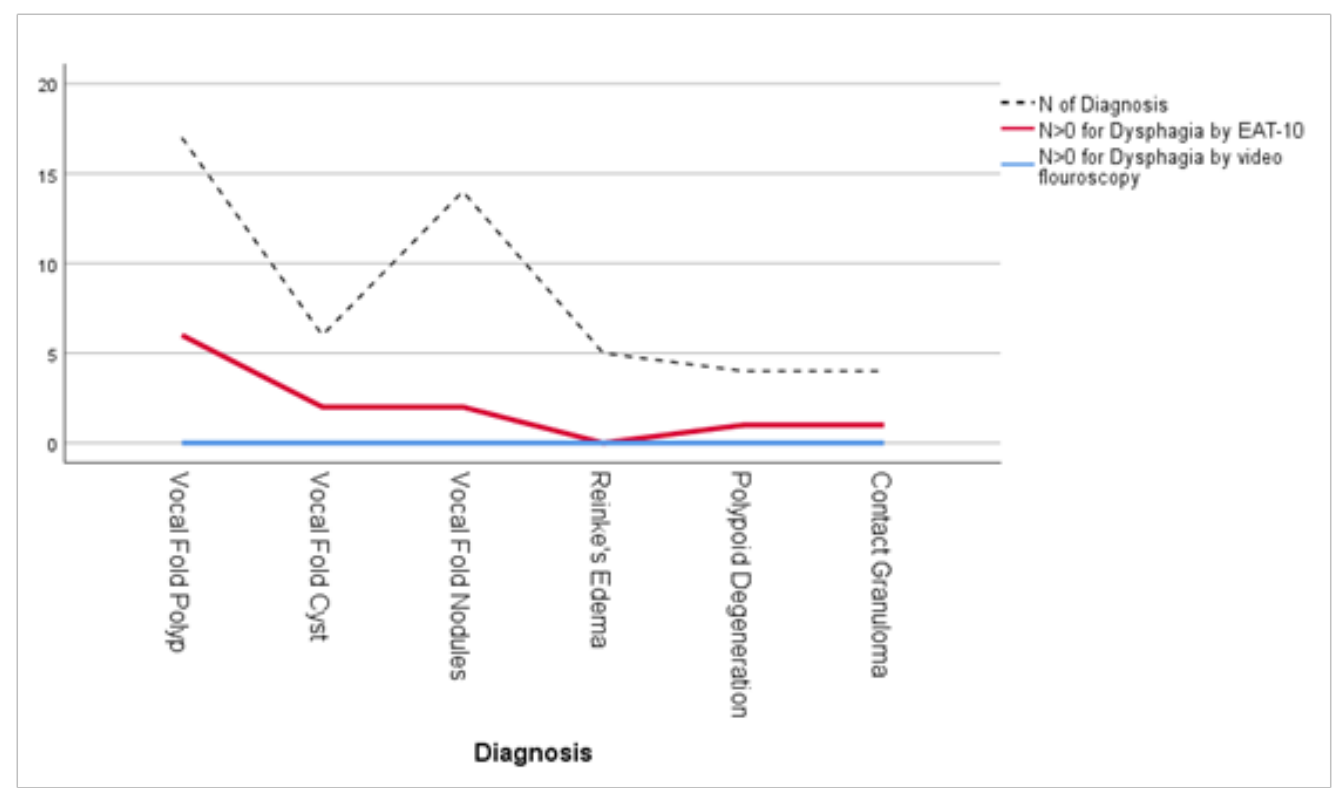

Fig. 1: Frequency of dysphagia by the Arabic EAT-10 and by Videoflouroscopy among each vocal fold lesion showing No correlation between both frequencies

\section{DISCUSSION}

Dysphagia is an important symptom that could have a huge impact on different aspects of a patient's life. Dehydration, aspiration pneumonia and malnutrition are of the clinical complications that may increase the mortality and morbidity rates as well as affecting the patients, quality of life and the patient's daily activities ${ }^{[6]}$.

Limited studies were done to search for the cause behind complaints of dysphagia reported by patients with dysphonia with minimal associated pathological lesions, whose vocal complaints may mask the symptoms of dysphagia.

The prevalence of dysphagia in patients with dysphonia secondary to minimal associated pathological lesions vocal fold lesions has not been previously reported in the literature except in only one study by Hamdan et al. ${ }^{[4]}$ uThe current study was done aiming at assessment of dysphagia symptoms in this group of patients relying on subjective and objective measures.

Among the different tools that detect dysphagia subjectively, the Arabic EAT-10 was chosen to be used in this study being a valid, reliable and rapidly noninvasive tool to measure patients' perception of their swallowing problems. Patients with a score above 3 were considered dysphagic. The results obtained in this study showed that $24 \%$ of the participating patients are dysphagic by the A-EAT-10, while Hamdan et al. ${ }^{[4]}$ showed that $37.7 \%$ of the patients are dysphagic by the A-EAT-10. Although subjectively by the A-EAT-10 patients report symptoms of dysphagia, additional objective measures are needed as diagnosis of dysphagia can't be confirmed depending only on subjective screening tools like the A-EAT-10, so in the current study VFSS was conducted on the patients proved to be dysphagic by the A-EAT-10 scoring above 3 . Among the 12 cases reported dysphagia by the A-EAT-10, the VFSS of two cases showed multiple swallows to clear the bolus of all food types and only one case showed mild vallecular residue with the bolus of all food types that was rapidly cleared. However, those mild findings are insignificant and not associated with aspiration, so we can't diagnose those patients as true dysphagic who need to be treated. The rest of the cases showed completely normal oral and pharyngeal phases of swallowing for all types of food by VFSS.

The results of the current study are reinforced by the fact that the Minimal Associated Pathological lesions (MAPLs) are benign, minimal, non-neoplastic and non-inflammatory 
lesions that have no threat to life, no associated pain and no serious loss of function. Their main impact is the affection of optimal use of voice in communication due to the presence of dysphonia ${ }^{[7]}$.

Also the intricacy in the neuromuscular supply of the pharynx and larynx can't explain dysphagia to be caused by MAPLs which are superficial lesions limited to the mucosa of the vocal folds and never extend beyond the mucosal level to affect the muscles or deeper structures of the larynx.

Hamdan et al. ${ }^{[4]}$ assumed that the pathophysiology of dysphagia in patients with dysphonia lies heavily on the intersection in the neuromuscular innervations of the upper airway and digestive system. In the pharyngeal phase of swallowing, there is anterior movement and elevation of the larynx, epiglottic closure, pharyngeal muscle contraction, and upper esophageal sphincter release, so a dysfunction in any of these stages may result in swallowing disorder. Other possible mechanisms were listed including restricted movement of the laryngeal framework, restricted pharyngeal constriction during swallowing, or alteration in the upper esophageal sphincter pressure during phonation. But lastly Hamdan et al. ${ }^{[4]}$ stated that these suggested mechanisms remain hypothetical in the absence of videofluoroscopic or manometric studies of swallowing.

On the highlight of the results of the current study, when a dysphonic patient secondary to MAPLs is complaining of dysphagia symptoms, it is mostly not related to his vocal fold lesions and the clinician should search thoroughly for another etiology.

It should be mentioned that patients with MAPLs have a functional element and may be suffering from associated phonasthenic symptoms that include symptoms related to throat as dryness, soreness, frequent clearance of the throat, sticky secretions of the throat difficult to be swallowed down, feeling of tightness or lump in the throat and rarely a feeling of ball in the throat "Globus"[7]. These symptoms may be misinterpreted by the patients as dysphagia.

\section{CONCLUSION}

The present study showed that non-neoplastic vocal fold lesions are not an etiological factor for dysphagia despite the intricacy in the neuromuscular supply of pharynx and larynx. Diagnosis of Dysphagia can't be confirmed depending only on subjective screening tools like the A-EAT-10.

\section{LIST OF ABREVIATIONS}

- Minimal associated pathological lesions (MAPLs)

- The Arabic-EAT-10 (A-EAT-10)

- Videoflouroscopic swallowing study (VFSS) -laryngo-pharyngeal reflux disease (LPRD)

\section{CONFLICT OF INTEREST}

There are no conflicts of interest.

\section{REFERENCES}

1. S.Y. Cho, R. S.Choungo, Y. A. Saito, C. D. Schleck, A. R.Zinsmeister, G. R. Locke III, N. J. Talley.Prevalence and risk factors for dysphagia: a USA community study, Neurogastroenterology and Motility. 27(2) (2015) 212-219.

2. T.Abou-Elsaad. Assessment and management of oropharyngeal dysphagia in adults: A workshop presented in the $27^{\text {th }}$ congress of International association of logopedics and phoniatrics, Copenhagen, Denmark, 2007.

3. N.A. Cipriani, D.E. Martin, J.P. Corey, L. Portugal, N. Caballero, R. Lester, B. Anthony, J.B. Taxy. Theclinicopathologic spectrum of benign mass lesions of the vocal fold due to vocal abuse. International journal of surgical pathology. 19(5) (2011) 583-587.

4. M.Farahat, T. A.Mesallam. Validation and cultural adaptation of the Arabic version of the Eating Assessment Tool (EAT-10). Folia Phoniatrica et Logopedica. 67(2015) 231-237.

5. A. L.Hamdan, E.Khalifee, H.Jaffal, A.Ghanem, S. A.Rizk, A. El Hage. Prevalence of Dysphagia in Patients with Non-neoplastic Vocal Fold Pathology. Journal of Voice33(5) (2019) 708-711.

6. C. L.Etges, B.Schreeren, E. Gomes, L.D. Barbosa. Screening tools for dysphagia: a systematic review. Communication disorders, Audiology and swallowing Journal26(5) (2014) 343-349.

7. M. N.Kotby, Y.AbouRas, M. Saleh, A.Khidr, M.Hegazi, A. El Adawy, M.Shadi. Non-organic voice disorders and the syndrome of vocal fatigue. Clinical Vocology 1(2016) 92-123.

8. Y. J.Jin, S. J. Lee, W. Y. Lee, W. J.Jeong, S. H. Ahn. Prognostic factors for prediction of follow-up outcome of contact granuloma. European Archives of OtoRhino-Laryngology 271(7) (2014) 1981-1985.

9. P. D.Karkos, M. George, J. Van Der Veen, H. Atkinson, R. C.Dwivedi, D. Kim, C. Repanos. Vocal process granulomas: a systematic review of treatment. Annals of Otology, Rhinology and Laryngology 123(5) (2014) 314-320.

10. S.W. Lee, H.J. Hong, S.H. Choi, D.I. Sun, Y.H. Park, 
B.J. Lee, S.K. Kwon, I.S. Park, S.H., Lee, Y.I. Son. Comparison of treatment modalities for contact granuloma: a nationwide multicenter study. The Laryngoscope 124(5) (2014),1187-1191.

11. R. Kobayashi, K.Tsunoda, R.Ueha, Y. Fujimaki,
T.Nito, T. Yamasoba. Role of lifestyle modifications for patients with laryngeal granuloma caused by gastro-esophageal reflux: comparison between conservative treatment and the surgical approach. Actaoto-laryngologica, 137(3) (2017) 306-309. 


\section{APPENDICES}

THE ARABIC-EAT-10 (A-EAT-10)

(Farahat and Mosallem, 2015).

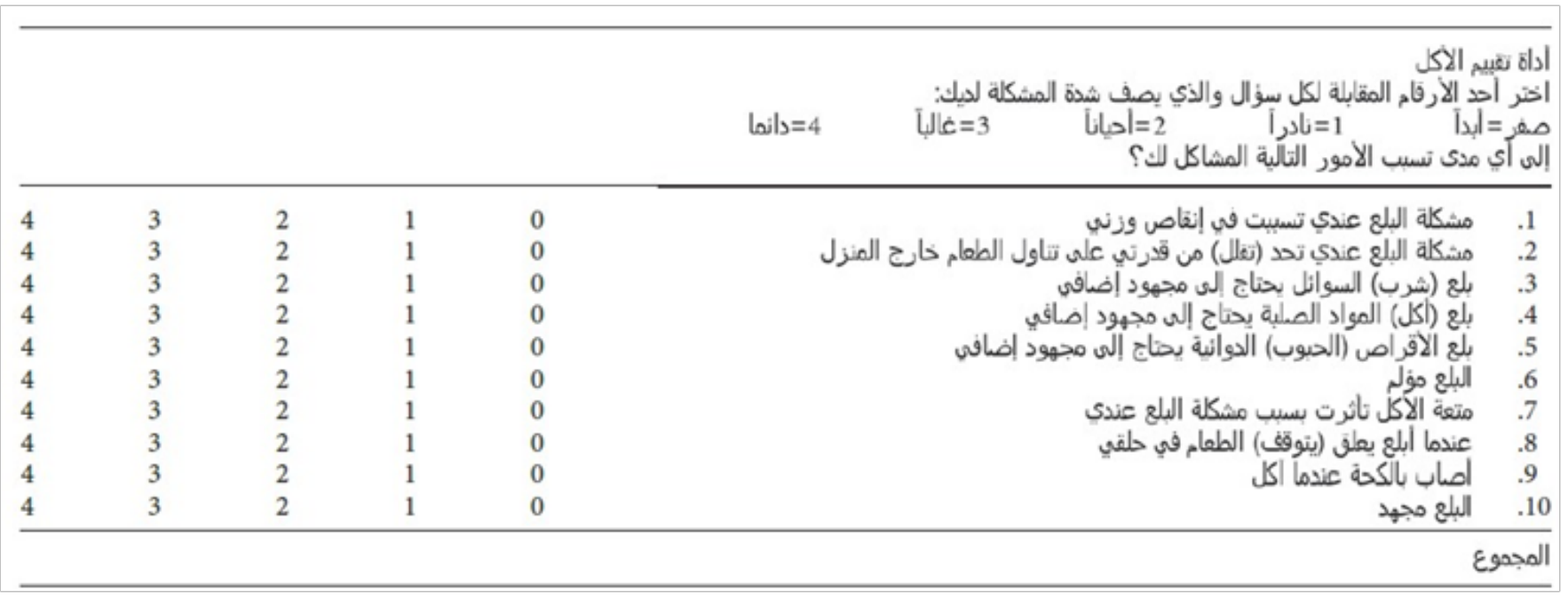

